\title{
INFLAMMATORY BREAST CANCER: A CLINICAL DIAGNOSIS
}

Singapore Med J 2014; 55(3): 170 doi:10.11622/smedj.2014043

I read the case report "Lactating breast abscess: a rare presentation of adenosquamous breast carcinoma" by Alipour et al ${ }^{(1)}$ with great interest. The authors presented an interesting case of breast cancer in a lactating woman who presented with a longstanding breast abscess of two months' duration. They have diligently reiterated the need to perform abscess wall biopsy in all atypical breast abscesses. They also ruled out the possibility of inflammatory breast cancer (IBC) on account of the absence of tumour emboli in the dermal lymphatics. However, I would like to stress that the presence of tumour emboli in the dermal lymphovascular spaces is neither sufficient nor required for the diagnosis of IBC.

IBC is clinically diagnosed by a short duration of symptoms, typical clinical signs on examination of the affected breast, and pathological diagnosis of breast carcinoma either through skin biopsy or core/needle biopsy of the underlying lump, if present. Rapidly progressing diffuse erythema (pink or mottled pink hue) and oedema of more than one-third of the breast distinguishes IBC from noninflammatory locally advanced breast cancer (LABC) with skin involvement. ${ }^{(2,3)}$ The breast oedema manifests as an increase in the size of the breast and exaggerated hair follicle pits, classically known as peau d'orange. These skin changes represent the dermal lymphatic obstruction, resulting from invasion by the tumour emboli; this phenomenon is considered a pathological hallmark of IBC.(4)

It is necessary to highlight here why dermal lymphatic invasion by tumour emboli is not considered diagnostic of IBC. Firstly, dermal tumour emboli may not be identified in all cases of IBC despite adequate sampling of the skin, and secondly, dermal tumour emboli, though less numerous and smaller in size, may also be seen in noninflammatory LABC. ${ }^{(2)}$ In a consensus statement, an international expert panel agreed that the following minimum criteria are required for the diagnosis of IBC: a history of rapid onset of breast erythema; a duration of history of no more than six months; oedema and/or peau d'orange and/or warm breast (with or without an underlying palpable mass); erythema occupying at least one-third of the breast; and pathological confirmation of invasive carcinoma. ${ }^{(4)}$ However, the panel also strongly recommended that skin biopsy be done in all patients suspected to have IBC, as it may confirm the presence of dermal tumour emboli and may also give the pathological diagnosis of carcinoma if no underlying lump or regional metastatic lymphadenopathy is detected.

A set of uniform diagnostic criteria for IBC, which can be used worldwide, will help medical practitioners obtain more accurate epidemiological data, and also provide better understanding of the clinicopathological profile and treatment outcomes of IBC across different geographical regions.

Yours sincerely,

Pankaj Kumar Garg

Department of Surgery, University College of Medical Sciences and Guru Teg Bahadur Hospital, University of Delhi, Dilshad Garden, Delhi 110095, India. dr.pankajgarg@gmail.com

\section{Conflict of Interest}

The views expressed in this letter are purely that of the author's and do not reflect the official position of the author's institution.

\section{References}

1. Alipour S, Seifollahi A, Anbiaee R. Lactating breast abscess: a rare presentation of adenosquamous breast carcinoma. Singapore Med J 2013; 54:e247-9.

2. Yamauchi H, Woodward WA, Valero V, et al. Inflammatory breast cancer: what we know and what we need to learn. Oncologist 2012; 17:891-9.

3. Robertson FM, Bondy M, Yang W, et al. Inflammatory breast cancer: the disease, the biology, the treatment. CA Cancer J Clin 2010; 60:351-75.

4. Dawood S, Merajver SD, Viens $\mathrm{P}$, et al. International expert panel on inflammatory breast cancer: consensus statement for standardized diagnosis and treatment. Ann Oncol 2011; 22:515-23. 